\title{
Association analysis of the polymorphism of human leukocyte antigen-A, -B and -E gene with Bechet's disease in Japanese cohort using sequencing-based typing method
}

\begin{abstract}
Behcet's disease (BD) is a chronic inflammatory autoimmune disease and strongly associated with human leukocyte antigen (HLA) gene such as $H L A-B$ and $-A$. To determine the association of the HLA-A, HLA-B and HLA-E alleles with BD in Japanese cohort as well as other cohorts, we performed sequencing-based typing method in $382 \mathrm{BD}$ cases and healthy controls. Then, we analyzed the association of these alleles with BD. Our results indicated that $H L A-B^{*} 51$ is strongly associated with $\mathrm{BD}$ in Japanese cohort (Allelic model's $\mathrm{P}$ value and OR are $4.59 \times 10^{-26}$ and 4.59 , respectively. Dominant model's $\mathrm{P}$ value and OR are $2.1 \times 10^{-24}$ and 5.32 , respectively), while $H L A-A^{*} 26$ is also significantly associated with BD (Allelic model's P value and OR are 5.3 $\times 10^{-5}$ and 1.80 , respectively. Dominant model's $\mathrm{P}$ value and $\mathrm{OR}$ are $8.6 \times 10^{-6}$ and 2.06 , respectively.). $H L A-E^{*} 01: 01$ was not observed to have any significant association with BD. We also obtained the available data sets of Chinese and Japanese genome sequence from public data base and then, analyzed each Linkage disequilibrium (LD) structure using SNPs in $H L A-A,-B$ and $-\mathrm{E}$ region. The pattern of LD including $H L A-E$ was different between two cohorts. Collectively, we suggested that $H L A-B * 51$ and $H L A-A * 26$ are associated with $\mathrm{BD}$ in Japanese cohort as well as another cohort and that $H L A-E^{*} 01: 01$ is not associated with $\mathrm{BD}$. Our data suggest that LD structure of the region including $H L A-E$ depends on a type of ethnic group even among Asian ethnic group.
\end{abstract}

Keywords: behcet's disease, human leukocyte antigen (HLA)-A, HLA-B, HLA-E, polymorphism, association study
Volume I Issue 2 - 2014

\author{
Riho Kurata,' Tomo Yonezawa, ${ }^{2}$ Hidetoshi \\ Inoko ${ }^{3}$ \\ 'Department of Experimental Animal Immunology, Tokyo \\ University of Science, Japan \\ 2Japan Society for the Promotion of Science, Japan \\ ${ }^{3}$ Department of Molecular Life Science, Tokai University School \\ of Medicine, Japan
}

\begin{abstract}
Correspondence: Riho Kurata, Department of Experimental Animal Immunology, Tokyo University of Science, 2669 Yamazaki, Noda, Chiba, Tel 8|-4-7|2|-4|05, Fax 8|-4-7|2|-4I05,
\end{abstract} Email yonet@rs.tus.ac.jp

Received: June 18, 2014 | Published: July 04, 2014
Abbreviations: $\mathrm{BD}$, behcet's disease; LD, linkage disequilibrium; HLA, human leukocyte antigen; GWAS, genomewide association studies; QC, quality control; OR, odds ratio

\section{Introduction}

Behcet's disease (BD) is a chronic inflammatory autoimmune disease characterized by recurrent oral and genital ulcers, skin legions and uveitis. ${ }^{1}$ T helper 1 (Th1) associated cytokines such as interferongamma and interleukin- 12 were enhanced in BD patients. ${ }^{2}$ Indeed, many studies have revealed the dominance of Th1 in the development of BD. ${ }^{3}$ Recently, it's been thought that another T cell subsets, Th17 plays an important role in the pathogenesis and development of BD. ${ }^{4}$ The incidence of $\mathrm{BD}$ is clearly high in the population who lives in from Middle East to Asia, along the "Old Silk Road", and rare in the USA especially in African Americans. ${ }^{5,6}$

The patient's family members have a predisposition of BD including genetic and environmental factors. Indeed, the incidence of BD is clearly higher in those peoples than in general peoples. ${ }^{7}$ Thus, epidemiological evidence indicates genetic factors contribute to the pathogenesis of BD. Several association studies have reported that human leukocyte antigen (HLA)- $B^{*} 51$ is strongly associated with $\mathrm{BD}$ among various ethnic groups including Japanese and Korean, ${ }^{8-11}$ while $H L A-A^{*} 26$ is also associated with BD. ${ }^{12,13}$ Among Korean cohort, $H L A-E^{*}$ 01:01 was shown to reduce the risk of BD. ${ }^{14}$ Except for HLA, we identified TRIM39 gene as novel susceptible gene of $\mathrm{BD}$ independently of $H L A-B * 51$ and $-A * 26 .{ }^{15}$. Recent Genome-wide association studies (GWAS) including our group identified that IL-
23R-IL12RB2 and IL-10 as novel BD susceptible loci. ${ }^{16,17}$ More recent study identified CCR1, STAT4 and KLRC4. ${ }^{18}$ These results support that Th1 and Th17 type inflammation have an important role in the pathogenesis and development of BD.

As well as in Korean cohort, whether or not $H L A-E^{*} 01: 01$ is associated with BD in Japanese cohort has remained to be identified. Thus, in this study, we performed sequencing-based typing of $H L A-A$, $-B$ and $-E$ using large amounts of genome sample from Japanese BD patients and healthy subjects. And then, we analyzed the polymorphism of each HLA and validated its association with BD susceptibility. We also obtained the data sets of genomic sequence including $H L A-A$, $-B$ and $-E$ in Japanese and Chinese cohort from public database and then, analyzed the difference of LD structure between two cohorts.

\section{Materials}

\section{Patients and controls}

We used a cohort of 384 Japanese unrelated BD patients and ethnically matched 384 unrelated healthy controls. All of the BD patients were diagnosed according to standard criteria. ${ }^{19}$ by the Japan Bechet's disease Research Committee at the Yokohama City University, Hokkaido University, Kurume University, Yuasa Eye Clinic and Fujioka Eye Hospital. These are included in our previous study. ${ }^{20}$ and. ${ }^{16}$. The study methodology compiled with the guidelines of the Declaration of Helsinki. The study details were explained to all case and controls before obtaining their consent for genetic screening. 


\section{Methods}

\section{Quality control filtering}

At first, quality control (QC) filtering of genotype data sets was performed to avoid false positive finding. We excluded two cases and two controls whose missing genotypes rates were more than 5\%. After that, we could not exclude any SNPs showing departure from HardyWeinberg equilibrium the data sets consisted of $382 \mathrm{BD}$ cases and 382 healthy controls were available. The OC filtering was performed by PLINK. ${ }^{21}$

\section{Genotype analysis of the HLA variants}

Genomic DNA was extracted from peripheral blood lymphocytes using QIAamp DNA Maxi Kit (Qiagen, Hilden, Germany) according to the manufacturer's instructions. To determine haplotypes of each HLA gene, we did sequencing-based typing method using primers previously reported in. ${ }^{22-24}$ Determined alleles were confirmed to be consistent with WHO Nomenclature 2006 ImMunoGeneTics/HLA Database (URL: http://www.ebi.ac.uk/ipd/imgt/hla/).

\section{Haplotype analysis}

We obtained data sets of Han Chinese in Beijing (CHB) and Japanese of Tokyo (JPT) genome from International Hap Map Project (URL: http://hapmap.ncbi.nlm.nih.gov) and then, visualized LD structure between two ethnic groups showing association signals Haploview. ${ }^{25}$ D' and R2 were used for measurement of LD. The significant threshold was $\left|\mathrm{D}^{\prime}\right|>0.8$ or R2 $>0.5$.

\section{Statistical analysis}

The statistical significance of the difference in each HLA allelic frequencies between $\mathrm{BD}$ patients and healthy controls was determined by the chi-square test and Fisher's exact test using PLINK. The levels of significance was set to that $P$ value is less than 0.05 , and odds ratios (OR) with $95 \%$ confidence intervals (CI) were calculated. We fit the data to three genetic models such as allelic, dominant and recessive.

Table I Association and analysis of HLA alleles with Behcet's disease

\begin{tabular}{|c|c|c|c|c|c|c|c|c|}
\hline $\mathrm{H}$ & $\mathbf{F r}$ & Fre & Allelic model & & Dominant moc & & Recessive moc & \\
\hline & $\mathbf{r} \mathbf{r}$ & rre & OR $\left(95 \%\right.$ Cl) ${ }^{\dagger}$ & P-value $\ddagger$ & OR (95\% CI) & $P$-value & OR (95\% CI) & $P$-value \\
\hline $\begin{array}{l}\text { HLA-A*26 } \\
\text { HLA-B*51 } \\
\text { HLA-E*01:01 }\end{array}$ & $\begin{array}{l}19.2 \\
28.4 \\
28.1\end{array}$ & $\begin{array}{l}11.7 \\
7.9 \\
31.6\end{array}$ & $\begin{array}{l}1.80(1.35-2.39) \\
4.59(3.38-6.23) \\
0.85(0.68-1.06)\end{array}$ & $\begin{array}{l}5.3 \times 10^{-5} \\
4.7 \times 10^{-26} \\
0.14\end{array}$ & $\begin{array}{l}2.06(1.50-2.83) \\
5.32(3.79-7.48) \\
0.78(0.59-1.04)\end{array}$ & $\begin{array}{l}8.6 \times 10^{-6} \\
2.1 \times 10^{-24} \\
0.111\end{array}$ & $\begin{array}{l}\text { I.2I }(0.37-4.00) \\
\text { NA } \\
0.90(0.56-1.46)\end{array}$ & $\begin{array}{l}0.77 \\
1.7 \times 10^{-8} \\
0.71\end{array}$ \\
\hline
\end{tabular}

†OR: Odds Ratio; $\mathrm{Cl}$ : Confidence Interval

$\ddagger$ The P-value was calculated using the Fisher's exact test.

\section{Results and discussion}

To examine whether or not $H L A-A,-B$ or $-E$ are associated with $\mathrm{BD}$ in Japanese cohort, we performed sequencing- based typing using large amount of BD patient and healthy control. In coincidence with previous reports, ${ }^{8-11} H L A-B^{*} 51$ was strongly associated with $\mathrm{BD}$ in Japanese cohort when we calculated in allelic model and dominant model (Table $1, P$ values are $4.59 \times 10^{-26}$ and $2.1 \times 10^{-24}$ respectively. OR are 4.59 and 5.32, respectively.), although we did not in recessive model. $H L A-A^{*} 26$ is also significantly associated with $\mathrm{BD}$ in both allelic model and dominant model (Table $1, \mathrm{P}$ values are $5.3 \times 10^{-}$ ${ }^{5}$ and $8.6 \times 10^{-6}$ respectively. OR are 1.80 and 2.06 , respectively.). However, $H L A-E^{*} 01: 01$ was not shown to have any significant association with $\mathrm{BD}$ even in three genetic models (Table 1). Park et al. ${ }^{14}$ reported that $H L A-E^{*} 01: 01$, which is one of the two major haplotypes, is significantly associated with BD in Korean cohort (P values and OR are 2.0x10-4 and 0.7, respectively). They also reported that the polymorphism of CD94/NKG2A, which are an inhibitory receptor complex binding to $H L A-E$, are associated with $\mathrm{BD}$ in Korean cohort. ${ }^{26}$ As such different susceptibility may be dependent on different genetic background of each cohort. To examine that, using available data sets from public database of human genome sequence, International HapMap Project, we also analyzed the difference of LD structure between JPT and CHB in the genomic region covering $H L A-A,-B$ and $-E$ (Figure 1).

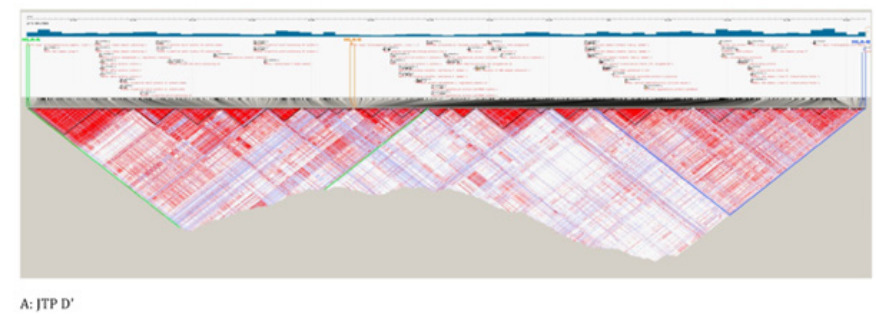

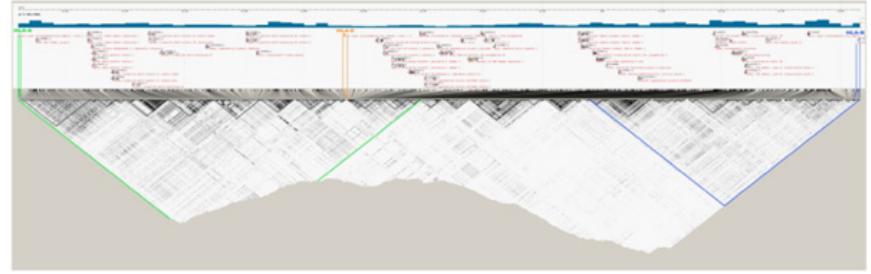

B: ITP R2

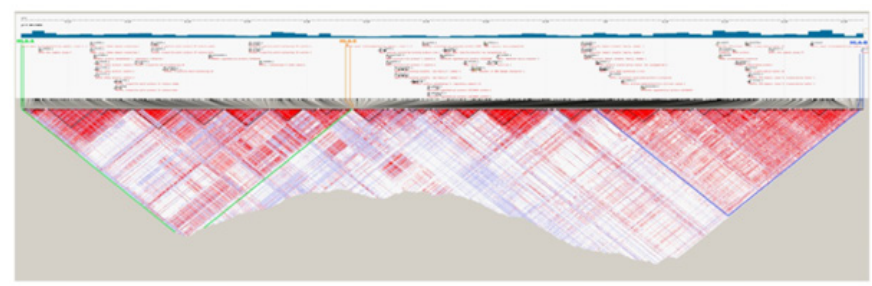

C: CHB D'

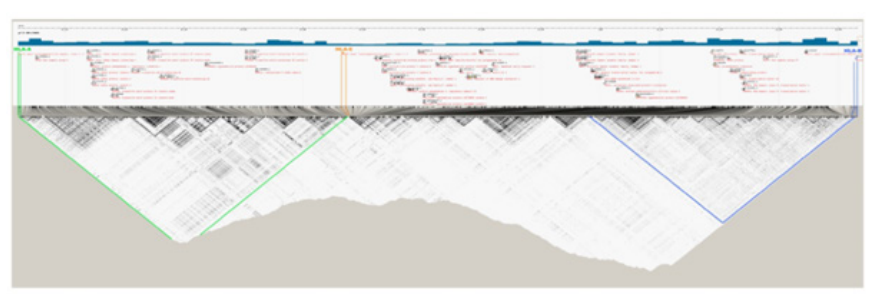

D: CHB R*

Figure I Regional association plot of HLA-A, $-B$ and -E region between JPT (A and $B$ ) and $C H B(C$ and $D$ ). A and $C$ indicate the measurement of $L D$ using $D$ ', while $B$ and $D$ indicate that of LD using R2. Light green line indicates HLA-A gene and its LD block, which possibly can affect other genome region. Light yellowish line indicates HLA-E gene. Light blue line indicates HLA-B and its LD block, which possibly can affect other genome region. 
Our result indicates that $\mathrm{LD}$ pattern of the region containing $H L A-E$ gene is different between JPT (Figures 1A \& 1B) and CHB (Figures 1C \& 1D). The pattern of LD in JPT was more associated with $H L A-A$ region than in CHB (Figure 1 light green and yellowish line). We hypothesized that susceptibility of HLA-E in JPT may be much higher than in CHB because JPT has stronger LD with $H L A-A$ (Figure 1 light green and yellowish line). Indeed, any $H L A-A$ allele is not significantly associated with BD in Han Chinese cohort, ${ }^{27}$ while it's associated in Japanese cohort. ${ }^{13}$ In contrast to $H L A-A$ region, those of $H L A-B$ were quite similar LD pattern between JPT and Chinese (Figure 1 Light blue line). These regions may be quite conserved among ethic groups. Taken together, we concluded that $H L A-B^{*} 51$ and $-A^{*} 26$ are associated with $\mathrm{BD}$ in Japanese cohort as well as another cohorts and that $H L A-E^{*} 01: 01$ is not associated with BD in Japanese cohort although previous study reported that it's associated with $\mathrm{BD}$ in Korean cohort. Our data suggest that LD structure of the region depends on a type of ethnic group even among Asian ethnic group, resulting in differences with susceptibility of HLA haplotype with BD.

\section{Acknowledgments}

This work was supported by Tokai University School of Medicine Research Aid, Health Labor Sciences Research Grant, Japanese Research Committee of Behcet's disease, Research on Measures for intractable Disease (No. 200), a Grant- $A$ id for JSPS Fellows (JSPS-12J07231). The authors thank Prof. Shigeaki Ohno (Hokkaido University Graduate School of Medicine) and Prof. Nobuhisa Mizuki (Yokohama City University School of Medicine) for their providing large amount of samples of BD patients.

\section{Conflicts of interest}

Author declares there are no conflicts of interest.

\section{Funding}

None.

\section{References}

1. Committee for the Preparation of Clinical Treatment of Ocular Lesions Related to Behcet's Disease. Guidelines for the clinical management of ocular Behcet's disease. Nihon Ganka Gakkai Zasshi. 2012;116(4):394426.

2. Frassanito MA, Dammacco R, Cafforio P, et al. Th1 polarization of the immune response in Behcet's disease:a putative pathogenetic role of interleukin-12. Arthritis Rheum. 1999;42(9):1967-1974.

3. Shimizu J, Yoshikawa $\mathrm{H}$, Takada E, et al. Unbalanced helper $\mathrm{T}$ cell function in Behcet's disease. Inflammation and Regeneration. 2011;31(3):296-301.

4. Hamzaoui K. Th17 cells in Behcet's disease:a new immunoregulatory axis. Clin Exp Rheumatol. 2011;29(4 Suppl 67):S71-S76.

5. Kaklamani VG, Vaiopoulos G, Kaklamanis PG. Behcet's Disease. Semin Arthritis Rheum. 1998;27(4):197-217.

6. Calamia KT, Wilson FC, Icen M, et al. Epidemiology and clinical characteristics of Behcet's disease in the US:a population-based study. Arthritis Rheum. 2009;61(5):600-604.

7. Kone-Paut I, Geisler I, Wechsler B, et al. Familial aggregation in Behcet's disease:high frequency in siblings and parents of pediatric probands. J Pediatr. 1999;135(1):89-93.

8. Horie Y, Meguro A, Kitaichi N, et al. Replication of a microsatellite genome-wide association study of Behcet's disease in a Korean population. Rheumatology (Oxford). 2012;51(6):983-986.
9. Piga M, Paladini F, Lai S, et al. Genetics of Behcet's disease in Sardinia:two distinct extended HLA haplotypes harbour the B*51 allele in the normal population and in patients. Clin Exp Rheumatol. 2012;30(3 Suppl 72):S51-S56.

10. Hamzaoui A, Houman MH, Massouadia M, et al. Contribution of Hla- $-B 51$ in the susceptibility and specific clinical features of Behcet's disease in Tunisian patients. Eur J Intern Med. 2012;23(4):347-349.

11. Mizuki N, Yabuki K, Ota M, et al. Analysis of microsatellite polymorphism around the $H L A-B$ locus in Iranian patients with Behcet's disease. Tissue Antigens. 2002;60(5):396-399.

12. Kang EH, Kim JY, Takeuchi F, et al. Associations between the $H L A-A$ polymorphism and the clinical manifestations of Behcet's disease. Arthritis Res Ther. 2011;13(2):R49.

13. Kaburaki T, Takamoto $M$, Numaga $J$, et al. Genetic association of $H L A-A^{*} 2601$ with ocular Behcet's disease in Japanese patients. Clin Exp Rheumatol. 2010;28(4 Suppl 60):S39-S44.

14. Park KS, Park JS, Nam JH, et al. HLA-E*0101 and HLA-G*010101 reduce the risk of Behcet's disease. Tissue Antigens. 2007;69(2):139144.

15. Kurata R, Nakaoka H, Tajima A, et al. TRIM39 and RNF39 are associated with Behcet's disease independently of $H L A-B^{*} 51$ and $-A * 26$. Biochem Biophys Res Commun. 2010;401(4):533-537.

16. Mizuki N, Meguro A, Ota M, et al. Genome-wide association studies identify IL23R-IL12RB2 and IL10 as Behcet's disease susceptibility loci. Nat Genet. 2010;42(8):703-706.

17. Remmers EF, Cosan F, Kirino Y, et al. Genome-wide association study identifies variants in the MHC class I, IL10, and IL23R-IL12RB2 regions associated with Behcet's disease. Nat Genet. 2010;42(8):698702

18. Kirino Y, Bertsias G, Ishigatsubo Y, et al. Genome-wide association analysis identifies new susceptibility loci for Behcet's disease and epistasis between $H L A-B^{*} 51$ and ERAP1. Nat Genet. 2013;45(2):202207.

19. Criteria for diagnosis of Behcet's disease. International Study Group for Behcet's Disease Lancet. 1990;335(8697):1078-1080.

20. Meguro A, Inoko H, Ota M, et al. Genetics of Behcet disease inside and outside the MHC. Ann Rheum Dis. 2010;69(4):747-754.

21. Purcell S, Neale B, Todd-Brown K, et al. PLINK:a toolset for wholegenome association and population-based linkage analysis. Am J Hum Genet. 2007;81(3):559-575.

22. Bunce $\mathrm{M}, \mathrm{O}$ 'Neill $\mathrm{CM}$, Barnardo MC, et al. Phototyping:comprehensive DNA typing for $H L A-A, \mathrm{~B}, \mathrm{C}, \mathrm{DRB} 1, \mathrm{DRB} 3, \mathrm{DRB} 4, \mathrm{DRB} 5 \& \mathrm{DQB} 1$ by PCR with 144 primer mixes utilizing sequence-specific primers (PCRSSP). Tissue Antigens. 1995;46(5):355-367.

23. Carter AS, Cerundolo L, Bunce M, et al. Nested polymerase chain reaction with sequence-specific primers typing for $H L A-A,-B$, and -C 37 alleles:detection of microchimerism in DR-matched individuals. Blood. 1999;94(4):1471-1477.

24. Grimsley C, Kawasaki A, Gassner C, et al. Definitive high resolution typing of $H L A-E$ allelic polymorphisms:Identifying potential errors in existing allele data. Tissue Antigens. 2002;60(3):206-212.

25. Barrett JC, Fry B, Maller J, et al. Haploview:analysis and visualization of LD and haplotype maps. Bioinformatics. 2005;21(2):263-265.

26. Seo J, Park JS, Nam JH, et al. Association of CD94/NKG2A, CD94/ $\mathrm{NKG} 2 \mathrm{C}$, and its ligand $H L A-E$ polymorphisms with Behcet's disease. Tissue Antigens. 2007;70(4):307-313.

27. Mineshita S, Tian D, Wang LM, et al. Histocompatibility antigens associated with Behcet's disease in northern Han Chinese. Intern Med. 1992;31(9):1073-1075. 